\title{
De-escalation of radiation dose for human papillomavirus-positive oropharyngeal head and neck squamous cell carcinoma: A case report and preclinical and clinical literature review
}

\author{
CHENG-CHIA WU ${ }^{1}$, DAVID P. HOROWITZ ${ }^{1}$, ISRAEL DEUTSCH ${ }^{1,2}$, RAHMATULLAH RAHMATI $^{3}$, \\ JORDAN M. SCHECTER ${ }^{4}$, ANJALI SAQI ${ }^{2,5}$ and TONY J. C. WANG ${ }^{1,2}$ \\ ${ }^{1}$ Department of Radiation Oncology, Columbia University College of Physicians and Surgeons; \\ ${ }^{2}$ Herbert Irving Comprehensive Cancer Center, Columbia University Medical Center; \\ Departments of ${ }^{3}$ Otolaryngology-Head and Neck Surgery, ${ }^{4}$ Medical Oncology, and ${ }^{5}$ Pathology and Cell Biology, \\ Columbia University College of Physicians and Surgeons, New York, NY 10032, USA
}

Received October 16, 2014; Accepted July 28, 2015

DOI: $10.3892 / 01.2015 .3836$

\begin{abstract}
Traditionally, head and neck squamous cell carcinoma (HNSCC) has been considered to be a relatively homogeneous disease. However, recent data have demonstrated that human papillomavirus (HPV)-positive and HPV-negative disease are two different clinical entities associated with different outcomes. Preclinical and clinical studies have reported a divergence in treatment strategies as well as prognostic outcomes for HNSCCs that are HPV-positive versus HPV-negative. The present study describes the case of a 52-year-old man who presented with stage IVB cT2N3M0 right tonsillar HPV-positive squamous cell carcinoma. Induction chemotherapy with docetaxel, cisplatin and 5-fluorouracil (TPF), followed by chemoradiation therapy with carboplatin and 70 Gray (Gy) radiation in daily fractions was recommended. The patient completed the TPF and carboplatin treatment; however, he was unable to tolerate the radiation course, receiving a final dose of 46 Gy. A 60-day follow-up right neck salvage dissection was subsequently performed. Despite having received a partial radiation treatment of $46 \mathrm{~Gy}$, the patient had no pathological evidence of disease at 60 days post radiation treatment. Repeat positron emission tomography-computed tomography at 32 months after the right neck dissection revealed no evidence of disease. The present study also discusses the current preclinical in vitro and in vivo targets for HPV-positive HNSCC and the obstacles presented in advancing
\end{abstract}

Correspondence to: Dr Tony J. C. Wang, Department of Radiation Oncology, Columbia University College of Physicians and Surgeons, North Basement, Room 11, 622 West 168th Street, New York, NY 10032, USA

E-mail: tjw2117@columbia.edu

Key words: human papillomavirus, de-escalation, radiation, oropharyngeal, head and neck squamous cell carcinoma clinical treatment modalities. Previous preclinical models investigating radiation sensitivity have yielded mixed results. Thus, it is important to understand and establish representative preclinical models for studying HPV and HNSCC to improve clinical research and therapeutic development. This review may guide future understanding of the role of HPV in HNSCC.

\section{Introduction}

Head and neck cancers represent the 7 th most common neoplasms worldwide (1). In 2012, there were an estimated 40,250 new cases in the United States and 7,850 mortalities secondary to these tumors (2), with the vast majority of head and neck cancers identified as squamous cell carcinomas (SCCs) by histology. Currently, treatment for patients with head and neck cancer involves multiple modalities, including chemoradiotherapy and/or surgery. The potential benefits of a chemoradiotherapeutic approach include functional organpreservation.

Traditionally, the selection of treatment modality for locally advanced head and neck squamous cell carcinoma (HNSCC) is based on tumor-related factors, including primary site, stage, patient characteristics and comorbidities (3). Patients with stage III-IV disease typically receive concurrent chemoradiotherapy of 70 Gray (Gy) in daily fractions with cisplatin administered every 3 weeks (4). Recently, there has been a growing interest in the role of human papillomavirus (HPV) in HNSCC pathogenesis (5) due to an associated rise in the incidence of HPV-positive oropharyngeal cancers, younger patient populations without the traditional head and neck cancer risk factors of tobacco and alcohol, and improved survival outcomes. These patients generally present with smaller primary tumors involving the palatine tonsils or base of tongue region, as well as multiple and/or large nodal metastases, giving them an advanced stage of disease at the time of presentation. As a result, patients may be treated with standard chemoradiation protocols that may lead to unnecessary long-term complications. The 
present study reports the case of a patient with HPV-positive right tonsillar SCC who received carboplatin-based combination chemotherapy and incomplete radiotherapy to a final tumor dose of $46 \mathrm{~Gy}$, with complete pathological response observed on post-chemoradiation surgical resection. Written informed consent was obtained from the patient. In addition, a literature review discussing the current preclinical studies associated with radiation sensitivity and HPV in HNSCC was performed.

\section{Case report}

In March 2012, a 52-year-old male with a history of daily marijuana use and no smoking history initially arrived at the Emergency Department of Columbia University Medical Center (New York, USA) with right-sided neck swelling that had lasted 1 week. The patient also exhibited mild odynophagia. Computed tomography (CT) of the neck was performed, revealing a large supraglottic mass involving the right tonsillar tissue as well as multiple neck lymphadenopathy, and a fine needle aspiration biopsy of the neck mass was completed for pathological assessment. Pathology revealed classic keratinizing SCC with necrosis and p16 positivity (Fig. 1). Hematoxylin and eosin staining revealed aty pical cells consistent with carcinoma (Fig. 1A and B). DiffQuick-stained smears showed squamous cells with dense cytoplasm and necrotic squamous cells (Fig 1C). Furthermore, Papanicolaou-stained smears showed neoplastic squamous cells with orangeophilic cytoplasm, consistent with keratinization (Fig. 1D). In addition, in situ hybridization staining for high risk HPV was positive (Fig. 1E). Positron emission tomography-computed tomography (PET-CT) was performed prior to treatment to assess the extent of the disease. This revealed a $6.7 \times 5.6 \times 10.8 \mathrm{~cm}$ right neck mass with maximal standardized uptake value (SUV) of 8.1, an inferior $3.7 \times 2.9 \mathrm{~cm}$ nodule with an SUV of 31.2 , and a right tonsillar mass measuring $3.9 \times 3.0 \mathrm{~cm}$ with an SUV of 23.4 (Fig. 2). Furthermore, scattered small bilateral neck lymph nodes were present, measuring $\leq 1.0 \times 1.0 \mathrm{~cm}$ with an SUV of 5.4. Considering the pathological confirmation of SCC and staging workup, the patient was diagnosed with stage IVB cT2N3M0 [AJCC TNM staging system (6)] right tonsillar HPV-positive SCC. A multidisciplinary tumor board discussion of the case resulted in the recommendation for induction chemotherapy with 3 cycles of docetaxel $\left(75 \mathrm{mg} / \mathrm{m}^{2}\right)$, cisplatin $\left(100 \mathrm{mg} / \mathrm{m}^{2}\right)$ and 5-fluorouracil $\left(1,000 \mathrm{mg} / \mathrm{m}^{2} ; \mathrm{TPF}\right)$, to be followed by definitive concurrent chemoradiation: External beam radiation for a total dose of 70 Gy (35 fractions of 2 Gy each) with concurrent chemotherapy of 7 cycles of carboplatin (7).

Following the completion of 3 cycles of induction TPF, a repeat PET-CT scan revealed a decrease in the size of the right neck mass to $3.6 \times 2.5 \times 7.7 \mathrm{~cm}$, with maximal SUV of 7.7 . Resolution of hypermetabolic activity was observed in the other cervical lymph nodes. Subsequently, the patient began concurrent chemoradiation therapy. However, due to persistent fatigue, he refused to complete the prescribed course of 70 Gy radiation, delivered via an intensity-modulated radiation therapy (IMRT) plan. The patient received a total of 46 Gy in 23 fractions over the course of 31 days. A repeat
PET-CT scan revealed a further decrease in the size of the right neck mass to $2.8 \times 2.8 \times 4.8 \mathrm{~cm}$, with a maximal $\mathrm{SUV}$ of 7.9 (Fig. 2).

At 60 days after the end of the shortened concurrent chemoradiation treatment, the patient underwent a salvage right neck dissection. Pathological findings revealed a $6.5 \times 4.0 \times 2.0 \mathrm{~cm}$ level II mass (8) that had extensive necrosis, fibrosis and mixed inflammatory infiltrate. No evidence of carcinoma was identified in 25 lymph nodes. The patient underwent no further treatment following the surgery. At 32 months after the right neck dissection, a repeat PET-CT revealed no evidence of disease, despite not receiving the full radiation. As there is no standard treatment for this disease entity, prognosis cannot be predicted.

\section{Discussion}

Classically, HNSCC has been considered to be a relatively homogeneous disease, and risk factors for HNSCC were predominantly thought to be environmental, including alcohol consumption and tobacco use $(9,10)$. The recommended treatment for stage III-IV HNSCC is typically concurrent chemoradiotherapy of 70 Gy in daily fractions with cisplatin delivered every 3 weeks (4). The role of HPV in HNSCC has become a growing area of research (5), with multiple preliminary studies suggesting that HPV status may have a significant impact on patient care and outcome $(11,12)$.

Ang et al (13), in a phase III clinical trial from the Radiation Therapy Oncology Group (RTOG 0129), first demonstrated that tumor HPV status is a strong and independent prognostic factor for survival among patients with oropharyngeal cancer. The findings from this study and others $(5,14)$ changed the understanding of HNSCC pathophysiology and treatment paradigms. It is now understood that HPV-positive and HPV-negative diseases are two very different clinical entities associated with vastly different outcomes $(5,14)$. Furthermore, over the last 10 years in the USA, there has been an increase in the incidence and survival of oropharyngeal cancers caused by $\operatorname{HPV}(15,16)$. As a result, new clinical trials, including the Eastern Cooperative Oncology Group (ECOG)-E1308 trial (17), have targeted HPV-positive HNSCC, with a focus on de-escalation of treatment based on the hypothesis that HPV-positive HNSCC requires a less aggressive treatment approach to achieve favorable outcomes (5). However, at present, little is known with regard to the molecular mechanisms underlying the increased sensitivity of HPV-positive HNSCCs to radiation, and there is a disconnect between preclinical research and clinical research addressing this topic. The following sections review preclinical and clinical research, discussing the role of HPV in radiation sensitization.

HPV in head and neck cancer and associated radiation sensitivity. The role of HPV in carcinogenesis was initially discovered by zur Hausen in cervical cancer (18). Since then, HPV has been extensively studied in cervical cancer and, more recently, in HNSCC (19-21). HPV and cancer radiation sensitivity has been extensively reviewed by Vozenin et al (22). In brief, ionizing radiation leads to the production of ionized molecules within the tissue. These molecules may then lead to series of effects that damage all cell molecules, most notably 

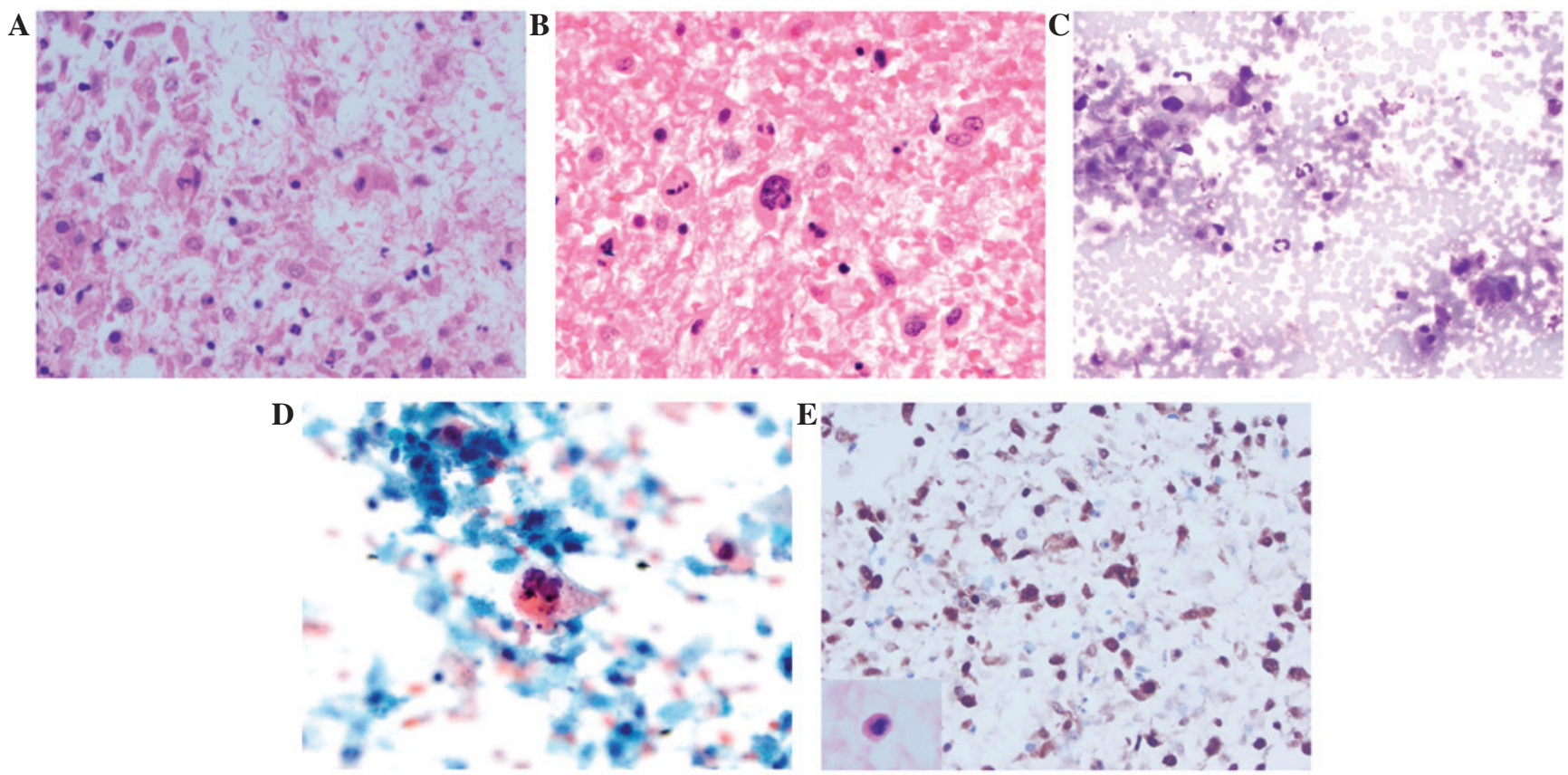

Figure 1. Tumor histopathology of the initial tumor biopsy. (A and B) Hematoxylin and eosin staining showing atypical cells consistent with carcinoma (magnification, x600). (C) Diff-Quik-stained smear showing squamous cells with dense cytoplasm and necrotic squamous cells (magnification, x400). (D) Papanicolaou-stained smear showing neoplastic squamous cells with orangeophilic cytoplasm, consistent with keratinization (magnification, x600). (E) p16 stain with an inset (left lower corner) showing a cell positive for high risk HPV by in situ hybridization (magnification, x600).
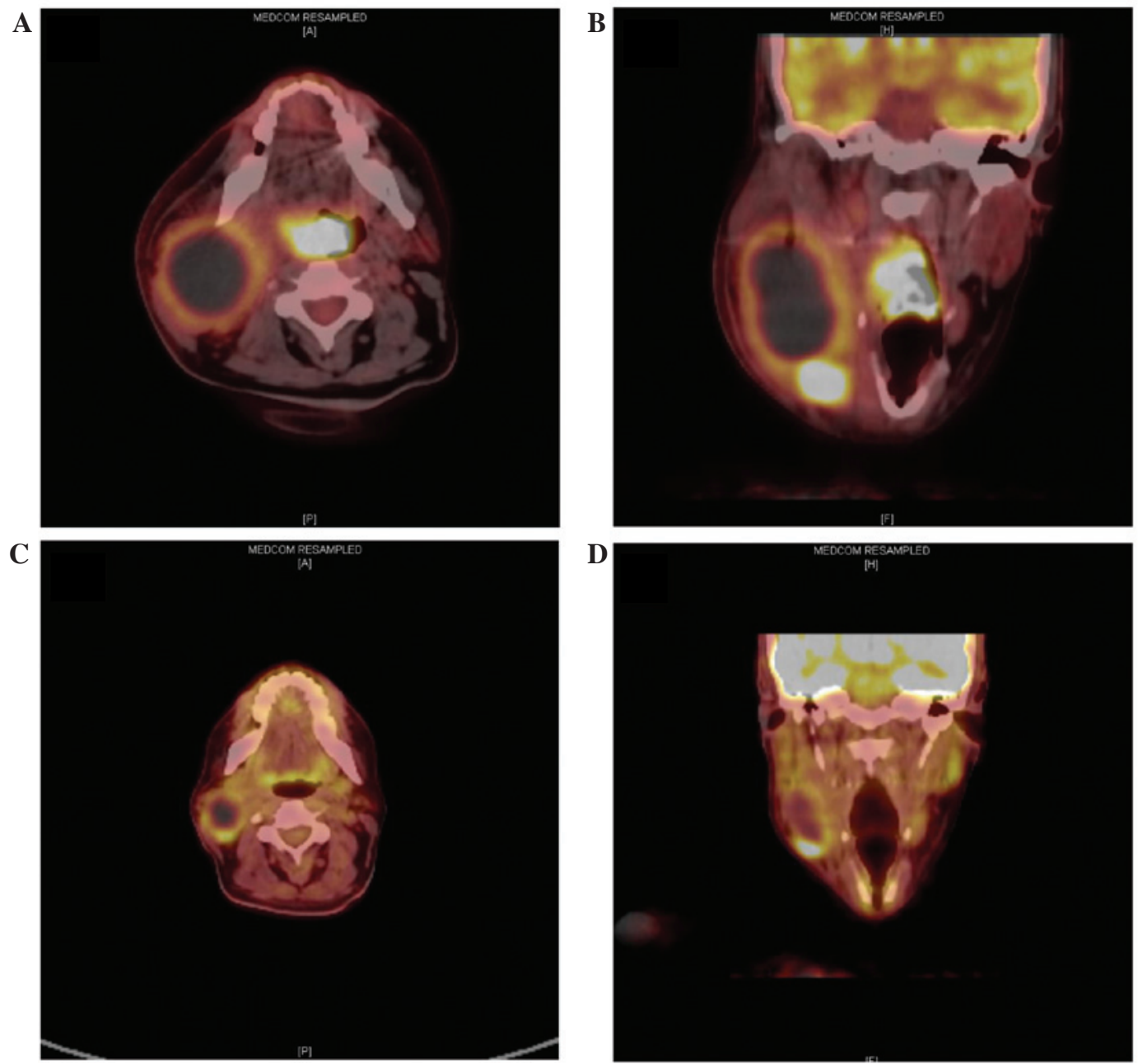

Figure 2. Pre-and post-treatment positron emission tomography-computed tomography images: (A) Pre-treatment axial section; (B) pre-treatment coronal section; (C) post-treatment axial section; (D) post-treatment coronal section. 
at the DNA level. Injuries to the DNA include base damages and single or double strand breaks (DSBs). The ability for a cell to sense these damages, repair the insult, halt cell cycle progression at various check points and initiate programmed cell death/apoptosis becomes important for cell radiosensitivity (22). In addition, further studies have demonstrated that manipulation of various cell signaling pathways, including epidermal growth factor receptor and phosphoinositide 3-kinase (PI3K)/protein kinase B (AKT)/mammalian target of rapamycin (mTOR) signaling, may also affect radiosensitivity $(22,23)$. Notably, tumor-type specific variations in radiosensitivity have been reported.

Gillison and colleagues first demonstrated a causal association between HPV and head and neck cancers (24). Since then, numerous studies have demonstrated the relationship between HPV status in HNSCC and radiotherapy (recently reviewed by Blitzer et al) (25). Traditionally, HPV has been hypothesized to alter radiation sensitivity through regulation of p53 and retinoblastoma (pRb) and downstream cell cycle control through viral E6 and E7, respectively. Cell cycle regulation and checkpoints are modulated by $\mathrm{p} 53$. Acting with cyclin-dependent kinases (CDKs) (cyclin/CDK4 and cyclin E/CDK2), p53 halts the cell cycle and facilitates the assessment of DNA integrity prior to cell division. In addition, p53 promotes apoptosis in severely damaged cells. HPV E6 manifests its function by causing degradation of p53, leading to aberrancy in cell cycle regulation and apoptosis. Similarly, $\mathrm{pRb}$ is also involved in cell cycle regulation. Unphosphorylated $\mathrm{pRb}$ binds to transcription factors, including $\mathrm{E} 2 \mathrm{~F}$ family members, and represses the transcription of genes involved in DNA synthesis and cell cycle progression, whilst phosphorylation leads to their activation. Binding of E7 to unphosphorylated $\mathrm{pRb}$ disrupts $\mathrm{pRb}-\mathrm{E} 2 \mathrm{~F}$ complexes, causing premature entry into $\mathrm{S}$ phase. Notably, one specific CDK inhibitor, $\mathrm{p} 16^{\mathrm{INK} 4 \mathrm{a}}$, is overexpressed when $\mathrm{pRb}$ is inactivated by HPV E7. Typically, overexpression of $\mathrm{p} 16^{\mathrm{INK} 4 \mathrm{a}}$ results in cell cycle arrest through promotion of pRb-E2F complexes; however, with HNSCC HPV E7-associated overexpression, p16 $6^{\text {INK4a }}$ has been used as biomarker for the evaluation of HPV status $(26,27)$. Inhibition of these two tumor suppressor genes (p53 via E6 or pRb via E7) leads to a failure to delay cell cycle progression. Furthermore, E6 and E7 contribute to cellular immortality through other mechanisms $(26,27)$. This leads to genetic instability following irradiation and may increase tumor resistance $(22,27)$.

However, the opposite is thought to be true in HPV-positive HNSCC, which may have a higher sensitivity to radiation compared with HPV-negative HNSCC $(24,28,29)$. There are numerous differences between HPV-positive and -negative HNSCC, including histological and clinical features, which were elegantly reviewed by Ang and Sturgis (30). Further molecular studies have demonstrated that HPV-positive HNSCCs are genetically and molecularly distinct from HPV-negative tumors. Common genetic aberrations for HNSCC, which include mutations in tumor protein p53 (TP53), CDK inhibitor 2A (CDKN2A; P16) and loss of heterozygosity at $3 \mathrm{p}$ and $17 \mathrm{p}$, are less common in HPV-positive HNSCC (26). Notably, Perrone and colleagues (31) examined the molecular and cytogenetic subgroups of HNSCC. In the groups that were HPV-positive, p53 mutation occurred in $12 \%$, and $100 \%$ of HPV-positive cases carried normal p16 ${ }^{\text {INK4a }}$. By contrast, $45 \%$ of the HPV-negative subgroup had p53 mutations, and $47 \%$ had p16 ${ }^{\text {INK4a }}$ homozygous deletion. The exact mechanism underlying the increased radiosensitivity of HPV-positive HNSCCs is unclear; however, the molecular and cytogenetic differences associated with positive and negative HPV status in HNSCC may contribute to the differences in response to therapy.

Preclinical studies in vitro. The findings of in vitro studies examining the effects of HPV on intrinsic tumor cellular radiation sensitivity have been unclear. Initial studies by Gupta et al (32) examined the response of two HPV-positive HNSCC cell lines (UPCI-SCC90 and UM-SCC47) and a HPV-negative line (SQ20B) to radiation therapy. Prior studies in cervical cancer had indicated that HPV-positive cervical carcinoma exhibited resistance to radiation treatment $(22,33,34)$. However, Gupta et al (32) demonstrated that the two HPV-positive HNSCC cell lines were more sensitive to radiation alone compared with HPV-negative cell lines. By contrast, Spanos et al reported that HPV-positive cells were more resistant to radiation compared with HPV-negative cells; however, in vivo, HPV-positive tumors were more sensitive to radiation (35). Subsequent studies by other groups using additional cell lines have produced mixed results. Most notably, Nagel et al (36) and others (37), examined four HPV-positive cell lines (UM-SCC-47, UD-SCC-2, UPCI-SCC90 and VU-SCC-147) and fourteen HPV-negative HNSCC lines, and observed no response differences to radiation. More recently, studies by Arenz et al (38) revealed that irradiated HPV-positive HNSCC cell lines progressed faster through S-phase, exhibiting an increase in accumulation in $\mathrm{G} 2 / \mathrm{M}$ phase as well as increased DNA DSBs. The increased radiosensitivity may be, in part, due to increased cell cycle dysregulation and impaired DNA DSB repair. Variations in findings may be due to the in vitro experimental parameters used to assess response to radiation, cell-line variability, p53 mutant status or the lack of systemic response following radiation treatment using in vitro models $(35,36,39)$. Furthermore, it is possible that the changes in radiosensitivity are not related to the intrinsic sensitivity of the tumor cells to radiation in vitro (35).

In order to address some of these variables, Kimple et al (39) performed single-cell isolation and clonal selection of four HPV-positive cell lines (UD-SCC-2, UM-SCC-47, UPCI-SCC90 and 93-VU-147T) as well as four HPV-negative cell lines (UM-SCC-1, UM-SCC-6, UM-SCC-22B and SCC-1483). HPV status was validated by Southern blot analysis and, using these validated clones, increased sensitivity to radiation in HPV-positive HNSCC lines compared with HPV-negative HNSCC lines was consistently shown, with clonogenic survival assays showing $22 \%$ vs. $59 \%$ survival following 2 Gy radiation treatment, respectively. This was further validated in vivo with cell line xenografts of the eight aforementioned cell lines, which were treated with a dose/fraction of $2 \mathrm{~Gy}$, delivered twice weekly to a total dose of 8 Gy. Under these conditions, none of the four HPV-negative groups exhibited a delay in tumor growth following irradiation compared with that of non-irradiated groups. However, three of the four HPV-positive cell lines (UD-SCC-2, UM-SCC-47 and UPCI-SCC90) did exhibit significant growth delays (39). 
Using clonal populations of HPV-positive tumors, these results suggest that HPV does indeed cause an intrinsic increase in radiosensitivity in HNSCC; however, it is still unclear whether molecular and cytogenetic variations in TP53, CDKN2A, and loss of heterozygosity at $3 p$ and $17 p$ further contribute to radiosensitivity.

In addition to altering radiation sensitivity alone, HPV status in HNSCC has been associated with differences in radiosensitization in response to various therapeutic agents. As described previously, HPV expresses E6 and E7 oncoproteins that bind to and neutralize the functions of p53 and $\mathrm{pRb}(26,27)$. The majority of HPV-positive HNSCCs have wild type p53, and agents targeting E6 and E7 function and/or expression in order to restore $\mathrm{p} 53$ and $\mathrm{pRb}$ function have been investigated as a way of increasing radiosensitivity. Cidofovir [(S)-1-(3-hydroxy-2-phosphonylmethoxypropyl)cytosine; 'HPMPC'] is an antiviral agent that has been demonstrated to exert antiproliferative effects on HPV infected cells (40). Abdulkarim et al (41) first examined the role of cidofovir on E6/E7 and downstream p53/pRb pathways in HNSCC using HEP2, a HPV-positive HNSCC cell line. The same group also determined whether cidofovir modulated radiosensitivity. Results revealed that cidofovir reduced E6/E7 and induced $\mathrm{p} 53 / \mathrm{pRb}$ in HEP2. A clonogenic survival assay of HEP2 cells treated with 2 Gy radiation and cidofovir demonstrated a marked reduction in survival compared with irradiation alone (2.5 vs. $28 \%$ ). Similar results were later demonstrated in HPV-positive HNSCC UPCI-SCC90 cells (42). Although targeting HPV using cidofovir appeared to be effective in increasing radiotherapeutic efficacy, reverse strategies activating downstream pathways triggered by HPV infections, have also been investigated. Pang et al (43) stably expressed whole E6 or specific splice isoforms of E6 in oropharyngeal SCC, demonstrating that these strategies confer radiosensitivity. Similarly, adenovirus-mediated $16^{\text {INK4a }}$ gene therapy in laryngeal SCC revealed that the combined adenovirus-p16 and radiation group exhibited greater inhibition of tumor growth, compared with any other treatment groups and controls (44). A recent study reported that E6 increases metabolic phenotype through mTOR by altering cellular metabolic function through pathways involved in hypoxia response element (45), and that upregulated mTOR activity in HNSCC is critical in local recurrence and survival. Rapamycin (sirolimus) is an mTOR inhibitor that prevents progression, growth, angiogenesis and lymphangiogenesis in HNSCC (45). The use of rapamycin concurrently with radiation has been shown to significantly enhance direct cell killing in a clonogenic assay using HPV-positive HNSCC cells in a dose-dependent manner (45).

Other strategies for modulating radiosensitivity, in addition to modulating HPV-mediated pathways, include hypoxic modifications by nimorazole; however, HPV-positive and -negative HNSCC displayed the same relative sensitization effect (46). Although various studies have investigated HPV status and methods to increase radiosensitivity in HNSCC, very little is known in terms of its mechanism. One possible mechanism includes the impairment of DSB repair capacity in HPV-positive HNSCC (37). Another model, proposed by Kimple et al (39), is that HPV-positive HNSCCs generally have a higher prevalence of wild type p53 compared with HPV-negative HNSCCs. E6 induces the degradation of p53, causing a low level of baseline p53; however, this low level of wild type p53 may be activated by radiation-induced DNA damage, resulting in partial arrest and increased cell death. This induction is not observed in HPV-negative HNSCC due to the high percentage of mutant p53 or alterations in p53 signaling. This was demonstrated by the upregulation of multiple TP53 pathway-related genes in HPV-positive cell lines following radiation. Complete knockdown of TP53 with siRNA resulted in radiation resistance in the same HPV-positive cell lines (39). In addition, an in vitro study demonstrated that the degree of Akt phosphorylation and activation is greater in HPV-negative versus HPV-positive HNSCC cells, and that Akt activation is inversely correlated with radiosensitivity (32). Furthermore, nelfinavir, a human immunodeficiency virus protease inhibitor able to downregulate Akt signaling, may further sensitize HNSCC cells to radiation in vitro (32).

A recent study by Gubanova et al (47) examined the role of SMG1, a PI3K-related kinase, with regard to HPV and HNSCC. SMG1 has been demonstrated to possess multiple functions, including regulation of mRNA with premature termination codons, maintenance of telomere integrity, regulation of cell lifespan, protection against apoptosis, oxidative stress resistance, embryogenesis, activation of p53 and irradiation-induced DNA damage and tumor suppressor roles (47). In respect to ionizing radiation, decreased SMG1 has been reported to increase radiation sensitivity in osteosarcoma (48). Gubanova et al (47) reported that the expression of SMG1 is diminished in HPV-positive SCCs through E6/E7-mediated SMG1 promoter hypermethylation. Furthermore, downregulation of SMG1 using shRNA in HPV-negative HNSCC reduced clonogenic survival upon radiation, whereas overexpression of SMG1 in HPV-positive HNSCC resulted in increased resistance to radiation (47). Currently, one limitation of preclinical studies on HPV and HNSCC in vitro is the availability of HPV-positive cell lines; these presently include UD-SCC-2 (hypopharynx), UPCI-SCC90 (base of tongue), UM-SCC-47 (lateral tongue), 93-VU-147T (floor of mouth), UM-SCC-14 (recurrent oral cavity) and HEP2 (larynx). Furthermore, certain studies have suggested that HPV-mediated effects on radiosensitivity are modulated by extrinsic factors, including an increased immune response $(35,45,49,50)$.

Preclinical studies in vivo. As described above, the available data regarding the increased radiation responsiveness of HPV-positive HNSCC in vitro has been mixed, despite clinical evidence of an association between HPV status and response rate. In order to further assess the role of HPV in HNSCC, further studies utilizing animal models with systemic responses are required. This is initially evident by the findings of a study by Spanos et al (35), as mentioned previously, in which HPV-positive tumors were more sensitive to radiation only in vivo, and not in vitro. This suggests that, in addition to the HPV-positive HNSCC intrinsic changes, the in vivo environment may also contribute to changes in radiation sensitivity. It has been postulated that radiation induces an antigenic immune response to HPV-positive HNSCC, leading to increased sensitivity (35). Further investigation demonstrated that radiation induces a dose-dependent reduction in CD47 expression and that the reduction in cancer cell CD47 expression increased phagocytosis via dendritic cells, thus 
increasing radiation-related immune clearance (49). To validate the role of the immune system in HPV-positive HNSCC radiosensitivity, a non-oncogenic HPV 16 E6/E7 adenoviral vaccine containing an E6/E7 mutant construct (rendering it non-oncogenic) was investigated for its ability to induce an HPV-specific immune response in preclinical mouse models and the subsequent effects of treatment with cisplatin and radiation were evaluated (50). Results from the study revealed that infection of tumor cells with the mutant E6/E7 construct did not affect $\mathrm{p} 53$ or $\mathrm{pRb}$ expression and telomerase activation was not altered. However, the mutant E6/E7 did induce an HPV-specific cell-mediated immune response, and a survival analysis of HPV-positive HNSCC treated with cisplatin and radiation revealed a significantly improved long-term survival versus the vector control group (50). Experiments studying the effects of rapamycin in HPV-positive HNSCC radiosensitization also demonstrated the importance of the immune response, as concurrent treatment with radiation and rapamycin prolonged survival time in immunocompromised mice; however, in immunocompetent mice, long-term tumor-free survival was increased by $21 \%$ (45).

Although much work is conducted in xenograft models using the various available cell lines for HPV-positive and -negative HNSCC, one drawback is the variation between cell lines from different sources. Attempts to minimize variation include clonal selection and characterization of the various cell lines (39); however, the use of immortalized cell lines has inherent limitations. Recent evidence suggests that immortalized cell lines from a variety of tumor types tend to have gene expressions that are more similar to each other than to their original tissue type $(51,52)$. These variations may limit the usage of this model for the accurate simulation of clinical conditions. To address this issue, Kimple et al (53) successfully established direct-from-patient tumorgrafts from 22 patients in athymic nude mice. Animals were irradiated with 2 Gy/fraction twice weekly for 4 weeks via an X-RAD 320 biologic irradiator, custom-designed to immobilize the animals and limit radiation exposure to the tumors of the dorsal flank. Results from the study revealed that 4 of 22 cases were positive for HPV 16 or HPV 18. Tumorgrafts responded well to radiation, exhibiting a 2-fold increase in time to tumor quadrupling. However, the comparison of dose response to radiation was not reported. These results establish a good preclinical model to further assess the role of dose de-escalation in the treatment of HPV-positive HNSCC (53).

Clinical studies relating to HPV, HNSCC and radiation. Since the late 1980s and early 1990s, there has been a growing interest in the role of HPV and HNSCC. An initial retrospective study by Haraf et al (54) examined 66 patients and tissue samples prior to primary treatment. HPV was positive in only $18 \%$ of the patients; however, a subset analysis of patients with stage IV disease revealed that HPV infection was correlated with overall survival time (54). Since then, extensive research has been conducted to examine the association between HPV and prognosis in HNSCC, including studies by Mellin et al (55), Gillison et al (24), Schwartz et al (56), Lindel et al (57), and Weinberger et al (58), all of which were extensively reviewed by Ang and Sturgis (30). In general, all of these studies consistently demonstrated that HPV-positive
HNSCC was associated with a decrease in relapse, recurrence, risk of cancer-specific mortality and all causes of mortality, and an increase in local control and disease-free and overall survival, to various degrees. A meta-analysis of the literature examining the association between HPV and overall survival and disease-free survival was published in 2007, and found that patients with HPV-positive HNSCC had a lower risk of mortality [meta hazard ratio (HR), 0.85 ; $95 \%$ confidence interval (CI), 0.7-1.0], and a lower risk of recurrence (meta HR, $0.62 ; 95 \% \mathrm{CI}, 0.5-0.8)$ compared with that of HPV-negative HNSCC patients (59).

These promising findings lead to prospective clinical trials examining the correlation between HNSCC and HPV status beginning with the ECOG 2399 trial funded by the US National Cancer Institute (ClinicalTrials.gov Identifier: NCT00014118). The ECOG 2399 trial was a phase II trial in which patients received two cycles of induction chemotherapy with intravenous paclitaxel $\left(175 \mathrm{mg} / \mathrm{m}^{2}\right.$; intravenous infusion over $3 \mathrm{~h}$ ) and carboplatin (area under the curve, 6) on day 1 of a 3 -week cycle, followed by concomitant paclitaxel $\left(30 \mathrm{mg} / \mathrm{m}^{2}\right.$ weekly; intravenous) and standard-fractionation external beam radiation therapy (70 Gy in 35 fractions over 7 weeks). The results of the study were analyzed by Fakhry et al (29) and HPV status was determined via the detection of HPV 16 DNA using in situ hybridization and p16 immunohistochemistry. Patients with HPV-positive HNSCC were found to have higher rate of response to induction chemotherapy ( 82 vs. $55 \% ; \mathrm{P}=0.01$ ) and chemoradiation therapy ( 84 vs. $57 \%$; $\mathrm{P}=0.007$ ), and an improved 2-year overall survival rate after a median follow-up of 39.1 months (95 vs. $62 \%$; $\mathrm{P}=0.005$, log-rank test) compared with that of HPV-negative patients. Furthermore, patients with HPV-positive HNSCC had a reduced risk of progression (HR, 0.27; 95\% CI, 0.10-0.75) and mortality from any cause (HR, 0.36; 95\% CI, 0.15-0.85) compared with HPV-negative HNSCC patients (29).

This eventually led to a pivotal study by Ang et al (13) that analyzed HNSCC tumor specimens from a large phase III trial, the RTOG 0129 study, to assess the effect of tumor HPV status on survival among patients with oropharyngeal SCC. In brief, patients with pathologically confirmed stage III or IV SCC of the oral cavity, oropharynx, hypopharynx or larynx without distant metastases were randomly assigned to receive high-dose cisplatin $\left(100 \mathrm{mg} / \mathrm{m}^{2}\right.$ body surface area) concurrently with accelerated-fractionation radiotherapy ( 72 Gy in 42 fractions over 6 weeks, with a concomitant boost of twice-daily irradiation for 12 treatment days) or standard-fractionation radiotherapy (70 Gy in 35 fractions over a 7-week period). Samples were assessed for HPV 16 DNA using in situ hybridization and for p16 expression via immunohistochemistry. HPV 16-negative tumors were further evaluated for the presence of additional oncogenic HPV types. The results revealed that $63.8 \%$ of patients had HPV-positive tumors, and that these patients had a higher 3-year overall survival rate compared with that of HPV-negative patients (82.4 vs. 57.1\%; $\mathrm{P}<0.001$, log-rank test), in addition to a $58 \%$ lower risk of mortality (HR, 0.42; 95\% CI, 0.27-0.66) following adjustment for age, race, tumor and nodal stage, tobacco exposure and treatment assignment (13).

Subsequently, additional clinical trials have been analyzed for the association between HPV and chemoradiation treatment outcomes; this includes studies by Rischin et al (Trans Tasman 
Table I. Clinical trials with dose de-escalation in head and neck squamous cell carcinoma.

$\begin{array}{lll}\text { Clinicaltrials.gov identifier } \quad \text { Phase } & \text { Description }\end{array}$

NCT01530997
NCT01716195

II

II

NCT01084083

II

NCT01525927

II

NCT01663259

II

NCT01932697

II

NCT01088802

II

NCT01706939

III

NCT01687413

III
Phase II study of de-intensification of radiation and chemotherapy for low-risk hpv-related oropharyngeal squamous cell carcinoma Phase II trial of induction chemotherapy followed by attenuated chemoradiotherapy for locally advanced head and neck squamous cell carcinoma associated with HPV

Phase II trial of induction chemotherapy followed by cetuximab (Erbitux ${ }^{\circledR}$ ) with low dose vs. standard dose intensity-modulated radiation therapy in patients with HPV-associated resectable squamous cell carcinoma of the oropharynx

Phase II trial of neoadjuvant chemotherapy for HPV-associated squamous cell carcinoma of the oropharynx followed by reduced dose radiotherapy/chemoradiotherapy for responders or standard dose chemoradiotherapy for non-responders

Reduced-intensity therapy for advanced oropharyngeal cancer in non-smoking HPV 16-positive patients

Phase II evaluation of adjuvant hyperfractionated radiation and docetaxel for HPV associated oropharynx cancer

A phase II study on treatment de-intensification in favorable squamous cell carcinoma of the oropharynx

The quarterback trial: A randomized phase III clinical trial comparing reduced and standard radiation therapy doses for locally advanced HPV 16-positive oropharynx cancer

Adjuvant De-escalation, Extracapsular spread, P16+, Transoral (A.D.E.P.T.) Trial for oropharynx malignancy

HPV, human papillomavirus.

Radiation Oncology Group 02.02 trial) (28), Posner et al (TAX-324 trial) (60) and Lassen et al (Danish Head and Neck Cancer Study Group 6 and 7 trials) (61), and results have been extensively reviewed $(30,62)$. Findings suggest that HPV-positive HNSCCs generally have more favorable prognosis in terms of local regional control, risk of progression and cancer-related and overall survival.

Current clinical guidelines for the treatment of HNSCC do not account for HPV status, despite the evidence indicating clinical differences between HPV-positive and -negative HNSCCs, including the improved outcomes associated with HPV status in HNSCC (63). Preclinical and clinical studies also indicate that HPV-positive HNSCCs are more responsive to radiation and chemoradiation treatments. The question remains whether patients with HPV-positive HNSCC may achieve similar results with reduced treatments, with the aim of preserving clinical outcome whilst minimizing the side effects associated with intensified treatment. Given the potential for long-term toxic effects from radiation, attempts to minimize its use have been investigated in various clinical trials. Marur et al (17) in the phase III ECOG 1308 trial examined the role of reduced radiation in HPV-positive oral pharyngeal SCC. Stage III and IVA-B resectable HPV-positive oral pharyngeal SCC were included. Patients received induction chemotherapy of paclitaxel (three treatments; $90 \mathrm{mg} / \mathrm{m}^{2}$ once per week), cisplatin (one treatment, $75 \mathrm{mg} / \mathrm{m}^{2}$ ) and cetuximab (one treatment, $\left.400 \mathrm{mg} / \mathrm{m}^{2}\right)$, followed by cisplatin $\left(250 \mathrm{mg} / \mathrm{m}^{2}\right.$ weekly; 3 cycles). Patients then received IMRT to a dose of $54 \mathrm{~Gy} / 27$ fractions with weekly cetuximab for patients with complete response, or standard therapy (69.3 Gy/33 fractions) with weekly cetuximab for those without a complete response. Preliminary results between March 2010 and October 2011 were presented at the American Society of Clinical Oncology 2013 meeting. Low dose IMRT/cetuximab was administered to $73.8 \%(59 / 80)$ of the patients [54 Gy (56 patients), 52 Gy (1 patient) or 40 Gy (2 patients)]. During chemoradiotherapy, oral mucositis $(31 \%)$, dysphagia $(17 \%)$ and radiation dermatitis $(8 \%)$ were observed in a proportion of the patients. According to the authors, it is premature to conclude the 2-year progression-free survival rate; however, a $\geq 85 \%$ progression-free survival rate will merit further study. In addition to ECOG 1308, numerous other clinical trials (Table I) are currently being conducted to examine whether HPV-positive HNSCC patients are able to achieve adequate clinical outcomes with reduced radiation treatment.

Future perspective. Oropharyngeal squamous cell carcinoma continues to increase in incidence, accounting for a plurality of HPV-associated cancers in the USA (64). Patients with HPV-positive tumors have an improved prognosis in comparison to those with HPV-negative tumors, leading to investigation into treatment de-escalation in favorable-risk patients. Additionally, HPV E6 and E7 present potential therapeutic targets for further improving outcomes in patients with 
HPV-positive tumors (65). Beyond the treatment of individual patients, public education measures and investigation of HPV vaccination in the prevention of HPV-associated oropharyngeal SCC have the potential to reduce the incidence of the disease (19).

Summary. The current case report describes a patient with HPV-positive HNSCC who received induction chemotherapy with TPF and concurrent chemoradiation with carboplatin and incomplete radiation treatment of 46 Gy via IMRT. Despite receiving a reduced radiation dose relative to the planned $70 \mathrm{~Gy}$, the patient exhibited no pathological evidence of disease at 2 months after the termination of treatment, and no evidence of disease at 32 months post right neck dissection. To date, the patient has not complained of any side effects associated with radiation. Follow-up to determine whether the decrease in radiation limited the side effect profile for the current patient would be of interest. In addition, further follow-up is required to determine whether the patient is in remission from his disease. This case supports the requirement to reexamine treatment strategies for HNSCC based on HPV status.

Current studies examining the role of HPV and HNSCC with respect to radiation sensitivity are limited. Although clinical evidence demonstrates an association between HPV and radiation sensitivity, little is known in terms of mechanistic causes for this effect; preclinical studies investigating the enhancement or inhibition of the HPV E6/E7 axis have reported improvements in radiation sensitivity. There are various obstacles preventing further understanding and optimization of treatment, including identifying the proper preclinical models for research. In addition, variations at the molecular and cytogenetic levels may add another level of complexity to the subgroups of HNSCC. Although there has been increasing interest in identifying prognostic markers for treatment, the question remains whether these targets may be used as therapeutic targets to further improve treatment. A notable study from the Washington University School of Medicine examined microRNA (miRNA) profiles in oropharyngeal SCC (66). In total, 150 oropharyngeal tumors were analyzed and six miRNAs were identified that were significantly associated with cancer survival. In particular, miR-142-3p, miR-146a and miR-26b were preferentially overexpressed in the surviving patients, while miR-31, miR-24 and miR-193b were overexpressed in the patients who succumbed to their disease. When assessing miRNA expression with respect to HPV expression, miR-31 had a 1.73-fold lower expression in HPV-positive SCC. The question remains whether targeting miR-31 may affect overall prognosis and radiation sensitivity. There must be greater effort in translational research, based on bench to bedside and back to bench, in order to help develop further treatment options.

\section{References}

1. Jemal A, Bray F, Center MM, Ferlay J, Ward E and Forman D: Global cancer statistics. CA Cancer J Clin 61: 69-90, 2011.

2. Siegel R, Naishadham D and Jemal A: Cancer statistics, 2012. CA Cancer J Clin 62: 10-29, 2012.

3. Brana I and Siu LL: Locally advanced head and neck squamous cell cancer: treatment choice based on risk factors and optimizing drug prescription. Ann Oncol 23 (Suppl 10): x178-x185, 2012.
4. Denis F, Garaud P, Bardet E, et al: Final results of the 94-01 french head and neck oncology and radiotherapy group randomized trial comparing radiotherapy alone with concomitant radiochemotherapy in advanced-stage oropharynx carcinoma. J Clin Oncol 22: 69-76, 2004.

5. Tribius S, Ihloff AS, Rieckmann T, et al: Impact of HPV status on treatment of squamous cell cancer of the oropharynx: What we know and what we need to know. Cancer Lett 304: 71-79, 2011.

6. Edge S, Byrd DR, Compton CC, Fritz AG, Greene FL and Trotti A (eds). AJCC Cancer Staging Manual. 7th edition. Springer-Verlag, New York, NY, 2010.

7. Posner MR, Hershock DM, Blajman CR, et al: Cisplatin and fluorouracil alone or with docetaxel in head and neck cancer. $\mathrm{N}$ Engl J Med 357: 1705-1715, 2007.

8. Grégoire V, Ang K, Budach W, et al: Delineation of the neck node levels for head and neck tumors: A 2013 update. DAHANCA, EORTC, HKNPCSG, NCIC CTG, NCRI, RTOG, TROG consensus guidelines. Radiother Oncol 110: 172-181, 2014.

9. Gandini S, Botteri E, Iodice S, et al: Tobacco smoking and cancer: A meta-analysis. Int J Cancer 122: 155-164, 2008.

10. Hashibe M, Brennan P, Benhamou S, et al: Alcohol drinking in never users of tobacco, cigarette smoking in never drinkers and the risk of head and neck cancer: pooled analysis in the international head and neck cancer epidemiology consortium. J Natl Cancer Inst 99: 777-789, 2007.

11. Friedman JM, Stavas MJ Cmelak AJ: Clinical and scientific impact of human papillomavirus on head and neck cancer. World J Clin Oncol 5: 781-791, 2014.

12. Chung CH and Schwartz DL: Impact of HPV-related head and neck cancer in clinical trials: Opportunity to translate scientific insight into personalized care. Otolaryngol Clin North Am 45: 795-806, 2012.

13. Ang KK, Harris J, Wheeler R, et al: Human papillomavirus and survival of patients with oropharyngeal cancer. N Engl J Med 363: 24-35, 2010.

14. O'Rorke MA, Ellison MV, Murray LJ, et al: Human papillomavirus related head and neck cancer survival: A systematic review and meta-analysis. Oral Oncol 48: 1191-1201, 2012.

15. Chaturvedi AK, Engels EA, Pfeiffer RM, et al: Human papillomavirus and rising oropharyngeal cancer incidence in the United States. J Clin Oncol 29: 4294-4301, 2011.

16. Stein AP, Saha S, Yu M, Kimple RJ and Lambert PF: Prevalence of human papillomavirus in oropharyngeal squamous cell carcinoma in the United States across time. Chem Res Toxicol 27:462-469, 2014

17. Marur S, Lee JW, Cmelak A, et al: A phase II trial of induction chemotherapy followed by cetuximab with low dose versus standard dose IMRT in patients with HPV-associated resectable squamous cell carcinoma of the oropharynx (OP). J Clin Oncol 30 (Suppl): ASCO Annual Meeting Abstract 5566, 2012.

18. zur Hausen H: Papillomaviruses in the causation of human cancers-a brief historical account. Virology 384: 260-265, 2009.

19. Wang MB, Liu IY, Gornbein JA and Nguyen CT: HPV-Positive oropharyngeal carcinoma: A systematic review of treatment and prognosis. Otolaryngol Head Neck Surg: Jun 29, 2015 (Epub ahead of print)

20. Jin L and Xu ZX: Recent advances in the study of HPV-associated carcinogenesis. Virol Sin 30: 101-106, 2015.

21. Ramakrishnan S, Partricia S and Mathan G: Overview of high-risk HPV's 16 and 18 infected cervical cancer: Pathogenesis to prevention. Biomed Pharmacother 70: 103-110, 2015.

22. Vozenin MC, Lord HK, Hartl D and Deutsch E: Unravelling the biology of human papillomavirus (HPV) related tumours to enhance their radiosensitivity. Cancer Treat Rev 36: 629-636, 2010.

23. Jaboin JJ, Shinohara ET, Moretti L, et al: The role of mTOR inhibition in augmenting radiation induced autophagy. Technol Cancer Res Treat 6:443-447, 2007.

24. Gillison ML, Koch WM, Capone RB, et al: Evidence for a causal association between human papillomavirus and a subset of head and neck cancers. J Natl Cancer Inst 92: 709-720, 2000.

25. Blitzer GC, Smith MA, Harris SL and Kimple RJ: Review of the clinical and biologic aspects of human papillomavirus-positive squamous cell carcinomas of the head and neck. Int J Radiat Oncol Biol Phys 88: 761-770, 2014.

26. Snow AN and Laudadio J: Human papillomavirus detection in head and neck squamous cell carcinomas. Adv Anat Pathol 17: 394-403, 2010.

27. Narisawa-Saito $M$ and Kiyono T: Basic mechanisms of high-risk human papillomavirus-induced carcinogenesis: Roles of E6 and E7 proteins. Cancer Sci 98: 1505-1511, 2007. 
28. Rischin D, Young RJ, Fisher R, et al: Prognostic significance of p16INK4A and human papillomavirus in patients with oropharyngeal cancer treated on TROG 02.02 phase III trial. J Clin Oncol 28: 4142-4148, 2010.

29. Fakhry C, Westra WH, Li S, et al: Improved survival of patients with human papillomavirus-positive head and neck squamous cell carcinoma in a prospective clinical trial. J Natl Cancer Inst 100: 261-269, 2008.

30. Ang KK and Sturgis EM: Human papillomavirus as a marker of the natural history and response to therapy of head and neck squamous cell carcinoma. Semin Radiat Oncol 22: 128-142, 2012.

31. Perrone F, Suardi S, Pastore E, et al: Molecular and cytogenetic subgroups of oropharyngeal squamous cell carcinoma. Clin Cancer Res 12: 6643-6651, 2006.

32. Gupta AK, Lee JH, Wilke WW, et al: Radiation response in two HPV-infected head-and-neck cancer cell lines in comparison to a non-HPV-infected cell line and relationship to signaling through AKT. Int J Radiat Oncol Biol Phys 74: 928-933, 2009.

33. Kamradt MC, Mohideen N and Vaughan AT: RU486 increases radiosensitivity and restores apoptosis through modulation of HPV E6/E7 in dexamethasone-treated cervical carcinoma cells. Gynecol Oncol 77: 177-182, 2000.

34. Liu S, Song L, Zhang L, Zeng S and Gao F: miR-21 modulates resistance of HR-HPV positive cervical cancer cells to radiation through targeting LATS1. Biochem Biophys Res Commun 459: 679-685, 2015.

35. Spanos WC, Nowicki P, Lee DW, et al: Immune response during therapy with cisplatin or radiation for human papillomavirus-related head and neck cancer. Arch Otolaryngol Head Neck Surg 135: 1137-1146, 2009.

36. Nagel R, Martens-de Kemp SR, Buijze M, et al: Treatment response of HPV-positive and HPV-negative head and neck squamous cell carcinoma cell lines. Oral Oncol 49: 560-566, 2013.

37. Rieckmann T, Tribius S, Grob TJ, et al: HNSCC cell lines positive for HPV and p16 possess higher cellular radiosensitivity due to an impaired DSB repair capacity. Radiother Oncol 107 242-246, 2013

38. Arenz A, Ziemann F, Mayer C, et al: Increased radiosensitivity of HPV-positive head and neck cancer cell lines due to cell cycle dysregulation and induction of apoptosis. Strahlenther Onkol 190: 839-846, 2014.

39. Kimple RJ, Smith MA, Blitzer GC, et al: Enhanced radiation sensitivity in HPV-positive head and neck cancer. Cancer Res 73 : 4791-4800, 2013.

40. Andrei G, Snoeck R, Piette J, Delvenne P and De Clercq E: Antiproliferative effects of acyclic nucleoside phosphonates on human papillomavirus (HPV)-harboring cell lines compared with HPV-negative cell lines. Oncol Res 10: 523-531, 1998.

41. Abdulkarim B, Sabri S, Deutsch E, et al: Antiviral agent Cidofovir restores p53 function and enhances the radiosensitivity in HPV-associated cancers. Oncogene 21: 2334-2346, 2002.

42. Sirianni N1, Wang J and Ferris RL: Antiviral activity of Cidofovir on a naturally human papillomavirus-16 infected squamous cell carcinoma of the head and neck (SCCHN) cell line improves radiation sensitivity. Oral Oncol 41: 423-428, 2005.

43. Pang E, Delic NC, Hong A, Zhang M, Rose BR and Lyons JG: Radiosensitization of oropharyngeal squamous cell carcinoma cells by human papillomavirus 16 oncoprotein E6*I. Int J Radiat Oncol Biol Phys 79: 860-865, 2011.

44. Fu YJ, Liu SX and Xian JM: Combination of adenovirus p16(INK4A) gene therapy and ionizing radiation for laryngeal squamous cell carcinoma. Sichuan Da Xue Xue Bao Yi Xue Ban 35: 209-211, 2004 (In Chinese).

45. Coppock JD, Wieking BG, Molinolo AA, et al: Improved clearance during treatment of HPV-positive head and neck cancer through mTOR inhibition. Neoplasia 15: 620-630, 2013

46. Sørensen BS, Busk M, Olthof N, et al: Radiosensitivity and effect of hypoxia in HPV positive head and neck cancer cells. Radiother Oncol 108: 500-505, 2013.

47. Gubanova E, Brown B, Ivanov SV, et al: Downregulation of SMG-1 in HPV-positive head and neck squamous cell carcinoma due to promoter hypermethylation correlates with improved survival. Clin Cancer Res 18: 1257-1267, 2012

48. Brumbaugh KM, Otterness DM, Geisen C, et al: The mRNA surveillance protein hSMG-1 functions in genotoxic stress response pathways in mammalian cells. Mol Cell 14: 585-598, 2004.

49. Vermeer DW, Spanos WC, Vermeer PD, et al: Radiation-induced loss of cell surface CD47 enhances immune-mediated clearance of human papillomavirus-positive cancer. Int J Cancer 133: 120-129, 2013.
50. Wieking BG, Vermeer DW, Spanos WC, Lee KM, Vermeer P, Lee WT, Xu Y, Gabitzsch ES, Balcaitis S, Balint JP Jr, et al: A non-oncogenic HPV 16 E6/E7 vaccine enhances treatment of HPV expressing tumors. Cancer Gene Ther 19: 667-674, 2012.

51. Gillet JP, Calcagno AM, Varma S, Marino M, Green LJ, Vora MI, Patel C, Orina JN, Eliseeva TA, Singal V, et al: Redefining the relevance of established cancer cell lines to the study of mechanisms of clinical anti-cancer drug resistance. Proc Natl Acad Sci USA 108: 18708-18713, 2011.

52. Uva P, Lahm A, Sbardellati A, Grigoriadis A, Tutt A and de Rinaldis E: Comparative membranome expression analysis in primary tumors and derived cell lines. PLoS One 5: e11742, 2010.

53. Kimple RJ, Harari PM, Torres AD, Yang RZ, Soriano BJ, Yu M, Armstrong EA, Blitzer GC, Smith MA, Lorenz LD, et al: Development and characterization of HPV-positive and HPV-negative head and neck squamous cell carcinoma tumorgrafts. Clin Cancer Res 19: 855-864, 2013.

54. Haraf DJ, Nodzenski E, Brachman D, Mick R, Montag A, Graves D, Vokes EE and Weichselbaum RR: Human papilloma virus and p53 in head and neck cancer: clinical correlates and survival. Clin Cancer Res 2: 755-762, 1996.

55. Mellin H, Friesland S, Lewensohn R, et al: Human papillomavirus (HPV) DNA in tonsillar cancer: clinical correlates, risk of relapse and survival. Int J Cancer 89: 300-304, 2000.

56. Schwartz SR, Yueh B, McDougall JK, Daling JR and Schwartz SM: Human papillomavirus infection and survival in oral squamous cell cancer: a population-based study. Otolaryngol Head Neck Surg 125: 1-9, 2001

57. Lindel K, Beer KT, Laissue J, Greiner RH and Aebersold DM. Human papillomavirus positive squamous cell carcinoma of the oropharynx: a radiosensitive subgroup of head and neck carcinoma. Cancer 92: 805-813, 2001.

58. Weinberger PM, Yu Z, Haffty BG, et al: Molecular classification identifies a subset of human papillomavirus-associated oropharyngeal cancers with favorable prognosis. J Clin Oncol 24: 736-747, 2006

59. Ragin CC and Taioli E: Survival of squamous cell carcinoma of the head and neck in relation to human papillomavirus infection: Review and meta-analysis. Int J Cancer 121: 1813-1820, 2007.

60. Posner MR, Lorch JH, Goloubeva O, et al: Survival and human papillomavirus in oropharynx cancer in TAX 324: A subset analysis from an international phase III trial. Ann Oncol 22: 1071-1077, 2011

61. Lassen P, Eriksen JG, Krogdahl A, et al: The influence of HPV-associated p16-expression on accelerated fractionated radiotherapy in head and neck cancer: Evaluation of the randomised DAHANCA 6\&7 trial. Radiother Oncol 100: 49-55, 2011.

62. Marur S, D'Souza G, Westra WH and Forastiere AA: HPV-associated head and neck cancer: a virus-related cancer epidemic. Lancet Oncol 11: 781-789, 2010.

63. National Comprehensive Cancer Network (NCCN): Clinical Practice Guidelines in Oncology. Head and neck cancer. Version 1, 2015. (Available at: http://www.nccn.org/professionals/physician_gls/pdf/head-and-neck.pdf). Accessed September 13, 2015.

64. Jemal A, Simard EP, Dorell C, Noone AM, Markowitz LE, Kohler B, Eheman C, Saraiya M, Bandi P, Saslow D, et al: Annual Report to the Nation on the Status of Cancer, 1975-2009, featuring the burden and trends in human papillomavirus (HPV)-associated cancers and HPV vaccination coverage levels. J Natl Cancer Inst 105: 175-201, 2013

65. Coordes A, Lenz K, Qian X, Lenarz M, Kaufmann AM and Albers AE: Meta-analysis of survival in patients with HNSCC discriminates risk depending on combined HPV and p16 status. Eur Arch Otorhinolaryngol: July 31, 2015 (Epub ahead of print).

66. Gao G, Gay HA, Chernock RD, Zhang TR, Luo J, Thorstad WL, Lewis JS Jr and Wang X: A microRNA expression signature for the prognosis of oropharyngeal squamous cell carcinoma. Cancer 119: 72-80, 2013. 